\title{
miR-15a and miR-16-1 downregulate CCND1 and induce apoptosis and cell cycle arrest in osteosarcoma
}

\author{
CHENG-KUI CAI $^{1 *}$, GUANG-YI ZHAO ${ }^{1 *}$, LI-YING TIAN $^{1}$, LIE LIU $^{2}$, KANG YAN $^{1}$, \\ YUN-LEI MA ${ }^{1}$, ZHEN-WEI JI ${ }^{1}$, XIAO-XIANG LI ${ }^{1}$, KANG HAN ${ }^{1}$, JIE GAO ${ }^{1}$, \\ XIU-CHUN QIU ${ }^{1}$, QING-YU FAN ${ }^{1}$, TONG-TAO YANG ${ }^{1}$ and BAO-AN MA ${ }^{1}$ \\ ${ }^{1}$ Department of Orthopedic Surgery Center and Orthopedic Oncology Institute of \\ People's Liberation Army, Tangdu Hospital, Fourth Military Medical University, Xi'an; \\ ${ }^{2}$ Department of Orthopedics, Baoji Center Hospital, Baoji, Shaanxi 710038, P.R. China
}

Received May 3, 2012; Accepted July 6, 2012

DOI: 10.3892/or.2012.1995

\begin{abstract}
Osteosarcoma, the most common primary tumor of the bones, causes many deaths due to its rapid proliferation and drug resistance. Recent studies have shown that cyclin D1 plays a key regulatory role during cell proliferation, and non-coding microRNAs (miRNAs) act as crucial modulators of cyclin D1 (CCND1). The aim of the current study was to determine the role of miRNAs in controlling CCND1 expression and inducing cell apoptosis. CCND1 has been found to be a target of miR-15a and miR-16-1 through analysis of complementary sequences between microRNAs and CCND1 mRNA. The upregulation of miR-15a and miR-16-1 in the cell line SOSP9607 induces apoptosis and cell cycle arrest. Osteosarcoma cells transfected with miR-15a and miR-16-1 show slower proliferation curves. Moreover, the transcription of CCND1 is suppressed by miR-15a and miR-16-1 via direct binding to the CCND1 3'-untranslated region (3'-UTR). The data presented here demonstrate that the CCND1 contributes to osteosarcoma cell proliferation, suggesting that repression of CCND1 by miR-15a and miR-16-1 could be used for osteosarcoma therapy.
\end{abstract}

\section{Introduction}

Osteosarcoma is a tumor characterized by the production of osteoid by malignant cells. This tumor commonly afflicts patients of 20 to 30 years of age, and the number has increased markedly in recent years. Tumor is a disease characterized by abnormal cell cycle phases. The first gap phase, G1, is unique

Correspondence to: Dr Tong-Tao Yang or Dr Bao-An Ma, Department of Orthopedic Surgery Center and Orthopedic Oncology Institute of People's Liberation Army, Tangdu Hospital, Fourth Military Medical University, Xi'an, Shaanxi 710038, P.R. China

E-mail: yangtt@fmmu.edu.cn; gukemba@fmmu.edu.cn

*Co-first authors

Key words: osteosarcoma, MiR-15a, MiR-16-1, crucial modulators of cyclin D1, apoptosis, cell cycle among cell cycle phases because it is the point at which cells are responsive to extracellular cues. Cyclin D1 plays a key regulatory role during the G1 phase and its gene is amplified and overexpressed in many cancers, such as non-small cell lung cancer (1), stomach cancer, and mantle cell lymphoma (2). However, the mechanism that would explain CCND1 deregulation in these cancer cells is yet to be discovered. MicroRNAs are a new class of small non-coding RNAs found in both animals and plants. They bind to the complementary sequences in the 3'UTR of the protein coding genes and induce mRNA degradation or translational repression (3). Growing evidence has indicated that microRNAs control basic cell functions, including development, differentiation, apoptosis, and proliferation $(4,5)$.

miR-15a and miR-16-1 are highly conserved RNAs that form a cluster at the chromosomal region 13q14.3 (6), which is frequently deleted in cancer. A number of studies have reported that miR-15a and miR-16-1 are missed or deleted in chronic lymphocytic leukemia (CLL), non-small cell lung carcinoma, liver cancer, breast cancer, ovarian cancer, prostatic cancer, stomach cancer, pituitary adenoma, multiple myeloma, and osteosarcoma (7-13). Numerous studies have reported that miR-15a and miR-16-1 induce apoptosis and inhibit cell proliferation by targeting multiple genes, but their specific mechanisms remain unclear.

In the current study, experimental evidence which shows that miR-15a and miR-16-1 induce apoptosis and cell cycle arrest in osteosarcoma was provided. In addition, a posttranscriptional regulatory mechanism of CCND1 expression mediated by miR-15a and miR-16-1 through direct interaction with the CCND1 mRNA at the 3'-UTR was discovered. CCND1 protein level expression is suppressed by miR-15a and miR-16-1, thus providing a potential strategy to prevent osteosarcoma proliferation by targeting the CCND1 oncogene.

\section{Materials and methods}

Cell lines. The human osteosarcoma cell lines SOSP-9607 and MG63 were grown in the RPMI-1640 medium and MEM medium with $10 \%$ heat-inactivated fetal bovine serum, $2 \mathrm{~g} / 1$ sodium bicarbonate, and $4 \mathrm{~g} / \mathrm{l} \mathrm{HEPES}$ (Sigma) at $37^{\circ} \mathrm{C}$ in an environment containing $5 \% \mathrm{CO}_{2}$. 
microRNA transfection. Cells were grown in the appointed medium 12-16 $\mathrm{h}$ before transfection. The cells were transfected with $100 \mathrm{~nm} / \mathrm{l}$ of miR-15a, miR-16-1, miR-15a inhibitor, miR-16-1 inhibitor, or negative control precursor using lipofectamine 2000 (Invitrogen) according to the protocol of the manufacturer. The microRNA mimics, inhibitors, and negative control precursor were from GenePharma. The efficiency of the transfection rate was measured using 5'-FAM marked negative control $5 \mathrm{~h}$ after transfection. The cells were harvested and processed for flow cytometry, cell cycle analysis, TUNEL, RT-PCR, immunocytochemistry, and western blot after $48 \mathrm{~h}$.

Apoptosis and cell cycle assays. Cultured cells were grown in 6-, 24-, and 96-well plates, respectively. They were divided into experimental groups, inhibitor groups, and control groups. The experimental groups consisted of the miR-15a and miR-16-1 groups. The inhibitor groups contained the miR-15a/miR-16-1 inhibitor groups, whereas the control groups contained negative and blank controls. Apoptosis and cell cycle were measured by flow cytometry. MTT was performed in $24,48,72$, and $96 \mathrm{~h}$, respectively. The absorbance at $492 \mathrm{~nm}$ was measured after incubation with $20 \mu \mathrm{l}$ MTT for $4 \mathrm{~h}$. The curve of cell proliferation was then drawn and the proliferation efficiency was examined. The in situ cell death detection kit (Roche Diagnostics, Indianapolis, IN, USA) was used for the TUNEL assay, and the procedures were performed as described by the manufacturer.

Target screening. In the current study, four publicly available search engines for target prediction were used: MiRanda, http://www.microrna.org (ref. 8505); PicTar, http://pictar.mdcberlin.de (ref. 746); TargetScan, http://genes.mit.edu/targetscan (ref. 968); and DIANA LAB, http://diana.cslab.ece.ntua.gr/ microT (ref. 684). The putative targets common to the different algorithms were obtained by sequentially inputting MiRanda hits into PicTar, followed by TargetScan, and finally into Diana lab. In accordance with other articles and repeated RT-PCR verifications, the CCND1 gene was finally chosen as the target.

RT-PCR and immunocytochemistry. RT-PCR and immunocytochemistry procedures were performed as described. Total RNA was extracted using TRIzol reagent (Invitrogen) according to the protocol of the manufacturer, and then transcribed into cDNA using BioRT Two Step RT-PCR kit (Bioer). The following primer set was used for RT-PCR: CCND1-F, 5'-CTGTG CATCTACACCGACAACT-3' and CCND1-R, 5'-GCATT TTGGAGAGGAAGTGTTC-3'. GAPDH was chosen as the normalization procedure. The primers were GAPDH-F, 5'-AGGTCCACCACTGACACGTT-3' and GAPDH-R, 5'-GCCTCAAGATCATCAGCAAT-3'. SP-0024 Histostain ${ }^{\text {TM }}$ Plus kits (BIOS) were used for immunocytochemistry. Mouse monoclonal (DCS-6) antibody to cyclin D1 antibody (Abcam) were utilized for immunocytochemistry (1:1000) and western blotting (1:200).

Western blot analysis. Protein extracts were prepared through a modified RIPA buffer with $0.5 \%$ sodium dodecyl sulfate (SDS) in the presence of proteinase inhibitor cocktail (Complete Mini, Roche Diagnostics). The procedure was as follows. Equal amounts of protein were resolved by $12 \%$ SDS-PAGE, then immunoblotted to the nitrocellulose membranes. The blocked membranes were soaked in 5\% evaporated skimmed milk for $2 \mathrm{~h}$, probed with primary antibody against cyclin D1 (Abcam) overnight at $4^{\circ} \mathrm{C}$, washed extensively with $20 \%$ Tween-20 in TBS, and incubated with goat anti-mouse monoclonal antibody conjugated with goat horseradish peroxidase (1:3000 dilution) for $2 \mathrm{~h}$. Mouse monoclonal anti- $\beta$-actin antibody (1:500 CWBIO) was used for normalization. The signals were visualized with enhanced chemiluminescence (Bio-Rad).

Luciferase report of CCND1. Through an analysis of the complementary sequences between the microRNAs and CCND1 mRNA, both miR-15a and miR-16-1 have been found to contain seven nucleotides that complement bases 1961 to 1967 of the CCND1 mRNA. Moreover, miR-15a has 12 nucleotides complementing bases 2033-2039, which is not a continuous complement. Apart from this finding, miR-16-1 has 11 nucleotides imperfectly complementing both bases 1961-1967 and 2033-2039, which facilitates the formation of the stem-loop structure (Fig. 4A). Thus, two 3'UTR segments of $195 \mathrm{bp}$ (1820-2014) and $265 \mathrm{bp}$ (1967 to2237) of the CCND1 gene were amplified by PCR from the total cDNA of SOSP9607 cells and inserted into the pMIR-REPORT ${ }^{\mathrm{TM}}$ Luciferase (pMIR-R-L) control vector (Ambion) using HindIII and SpeI as restriction sites. The primer set used to generate specific fragments are shown in Fig. 4D. Two inserts with deletions of $7 \mathrm{bp}$ (3'Ma) and 8 bp (3'Mb), respectively, were also generated from the perfect complementarity site using the QuikChange XL Site-Directed Mutagenesis kit (Stratagene). Target segments and mutant inserts were confirmed by sequencing. Renilla luciferase vector was used for normalization. The cells were co-transfected in 24-well plates using lipofectamine 2000 according to the protocol of the manufacturer with $0.2 \mu \mathrm{g}$ pMIR-R-L vector and $0.04 \mu \mathrm{g}$ control vector. Moreover, $100 \mathrm{~nm} / 1$ microRNA mimics or inhibitors were used for each well. pMIR-R-L and Renilla luciferase activities were measured consecutively using the dual-luciferase reporter assay system (Promega) $24 \mathrm{~h}$ after transfection.

Statistical analysis. All values were expressed as means \pm standard deviation (SD). Statistical significance was determined using one-way ANOVA for multiple comparisons and Student's t-test was used to compare two groups. $\mathrm{P}<0.05$ was considered significant.

\section{Results}

Apoptosis of human osteosarcoma cell line SOSP-9607 following transfection with miR-15a and miR-16-1. Two different assays were used to identify the biological effects of miR-15a and miR-16-1. Flow cytometry which was used to examine phosphatidylserine translocation showed that the cells transfected with miR-15a and miR-16-1 mimics obtain higher apoptosis ratio compared with the inhibitor and control groups (Fig. 1A and B, Q2 $\mathrm{P}<0.05$ ). There was no observed difference between the inhibitor and control groups (Fig. $1 \mathrm{~A}$ and B, P>0.05). TUNEL assay which was used to confirm DNA breakage and chromatin condensation showed that more apoptotic cells are significantly found in miR-15a and miR-16-1 groups (Fig. 1C left and $\mathrm{D}, \mathrm{P}<0.05)$, and no difference is found among the 

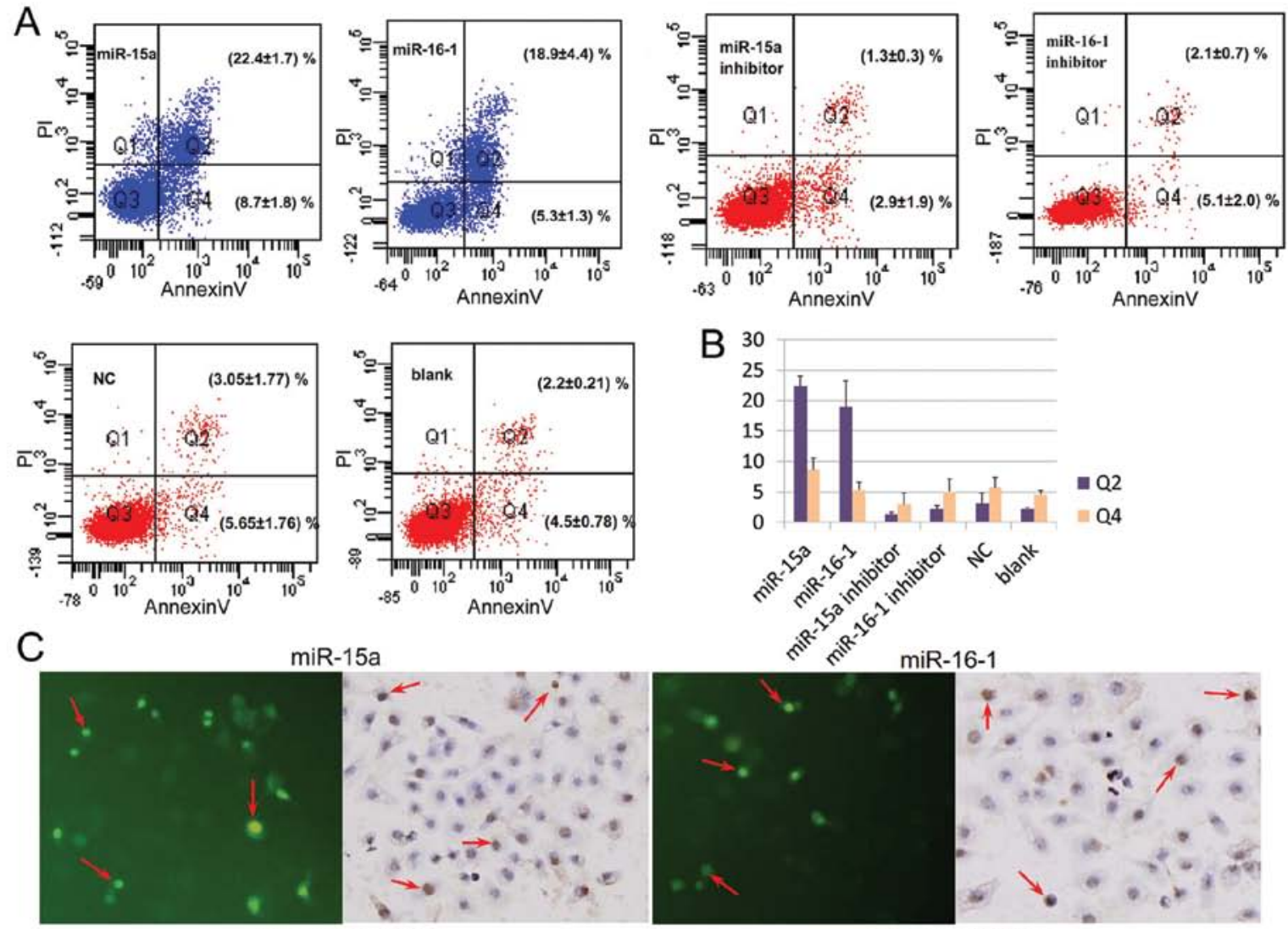

miR-15a inhibitor
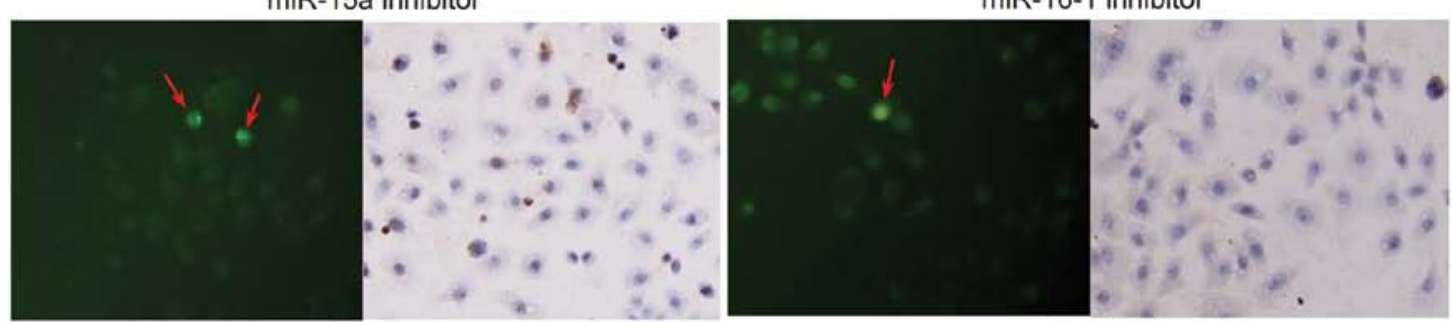

NC

blank
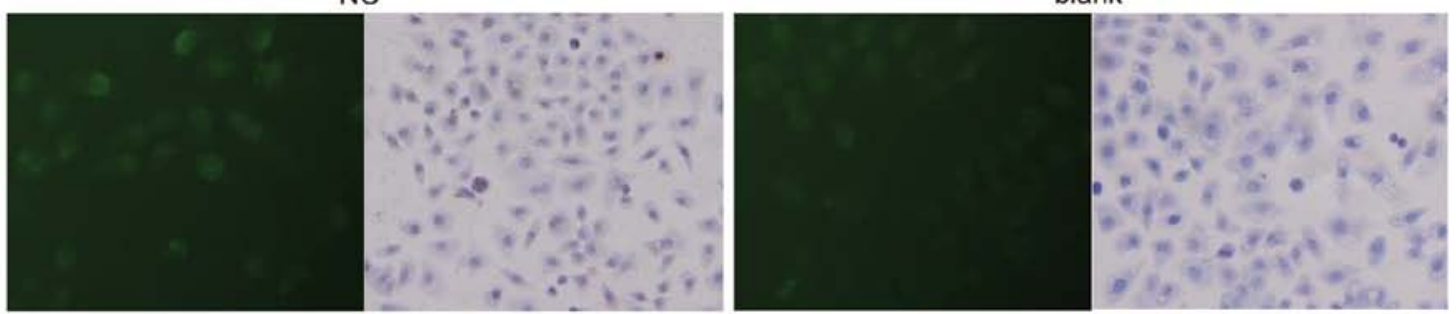

positive control
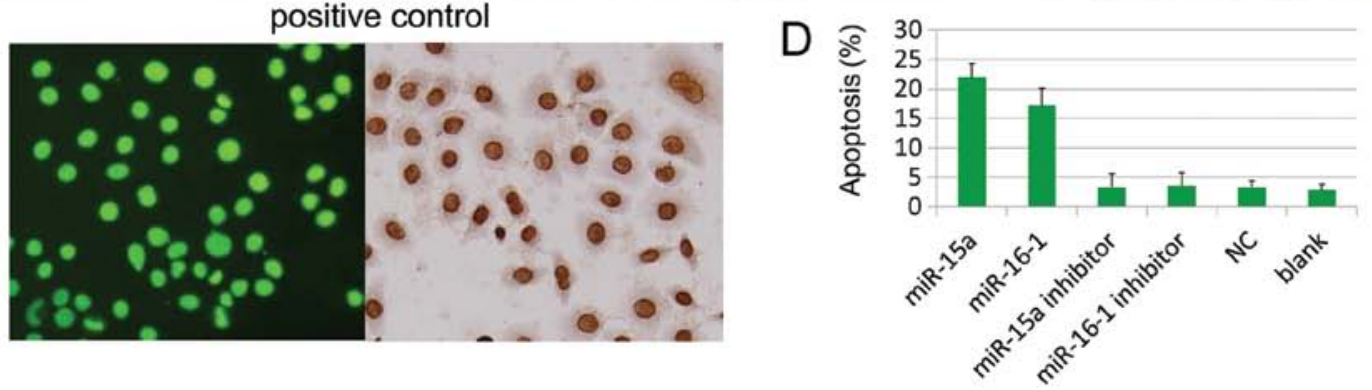

Figure 1. miR-15a and miR-16-1 induced apoptosis in human osteosarcoma cell line SOSP-9607. (A and B) Apoptosis ratio of SOSP-9607 cells $48 \mathrm{~h}$ after transfection with microRNA mimics. The data are the means \pm SD of four independent experiments. Cells in Q2 were either necrosis and/or late apoptosis with impaired membrane and phosphatidylserine translocation, whereas cells in Q4 were necrosis and/or early apoptosis with intact membrane and phosphatidylserine translocation. Based on one-way ANOVA and Student's t-test, the experiment groups have higher apoptosis ratios (Q2) than the inhibitor and control groups $(\mathrm{P}<0.05)$. No difference between inhibitor and control groups $(\mathrm{P}>0.05)$ has been observed. (C) TUNEL assay (left) and converter-POD/haematoxylin staining (right) showed significant apoptosis ratios between the experiment and other groups. The apoptosis cells (red arrows) are indicated in the images (magnification, x200). (D) Statistical analysis of apoptosis ratios in TUNEL. The data are the means \pm SD of five independent areas. The miR-15a and miR-16-1 groups obtained higher apoptosis ratios than the rest of the groups $(\mathrm{P}<0.05)$. 

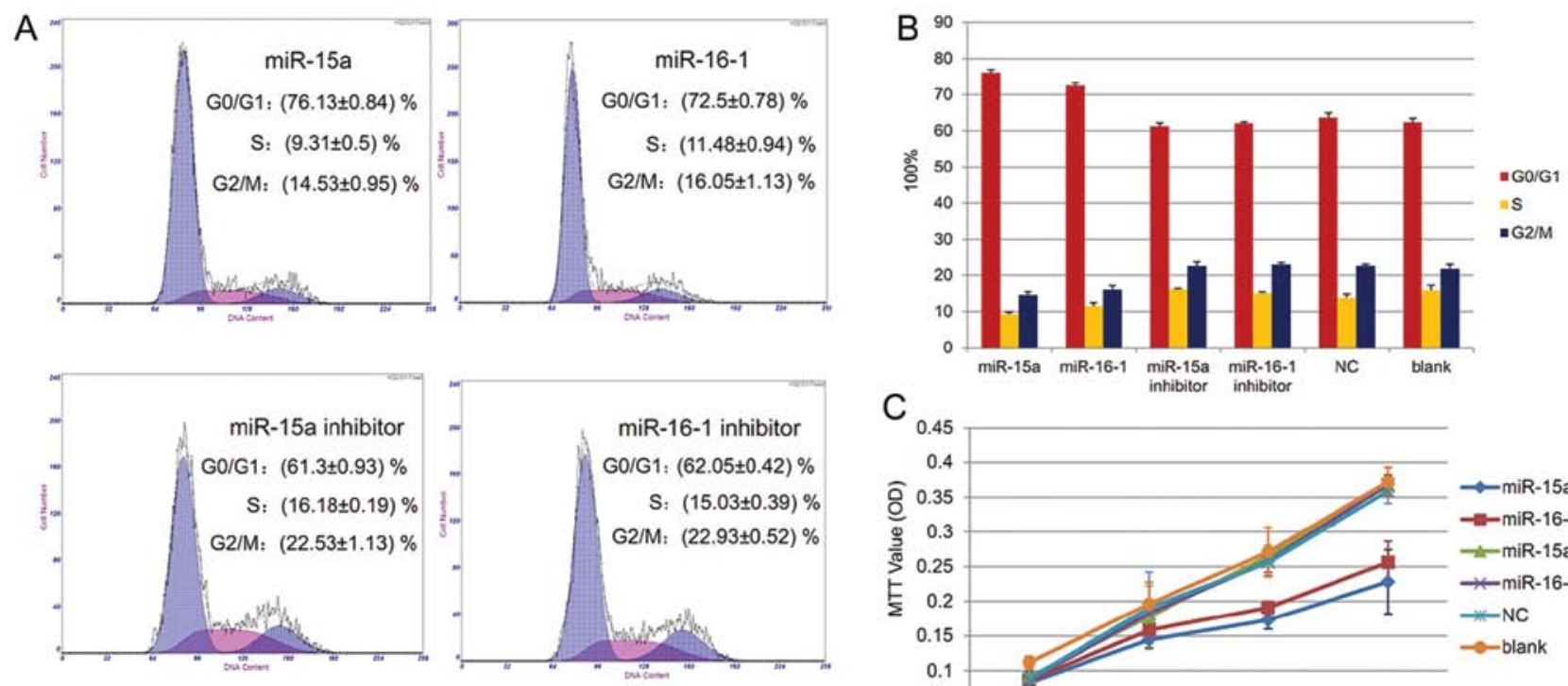

C
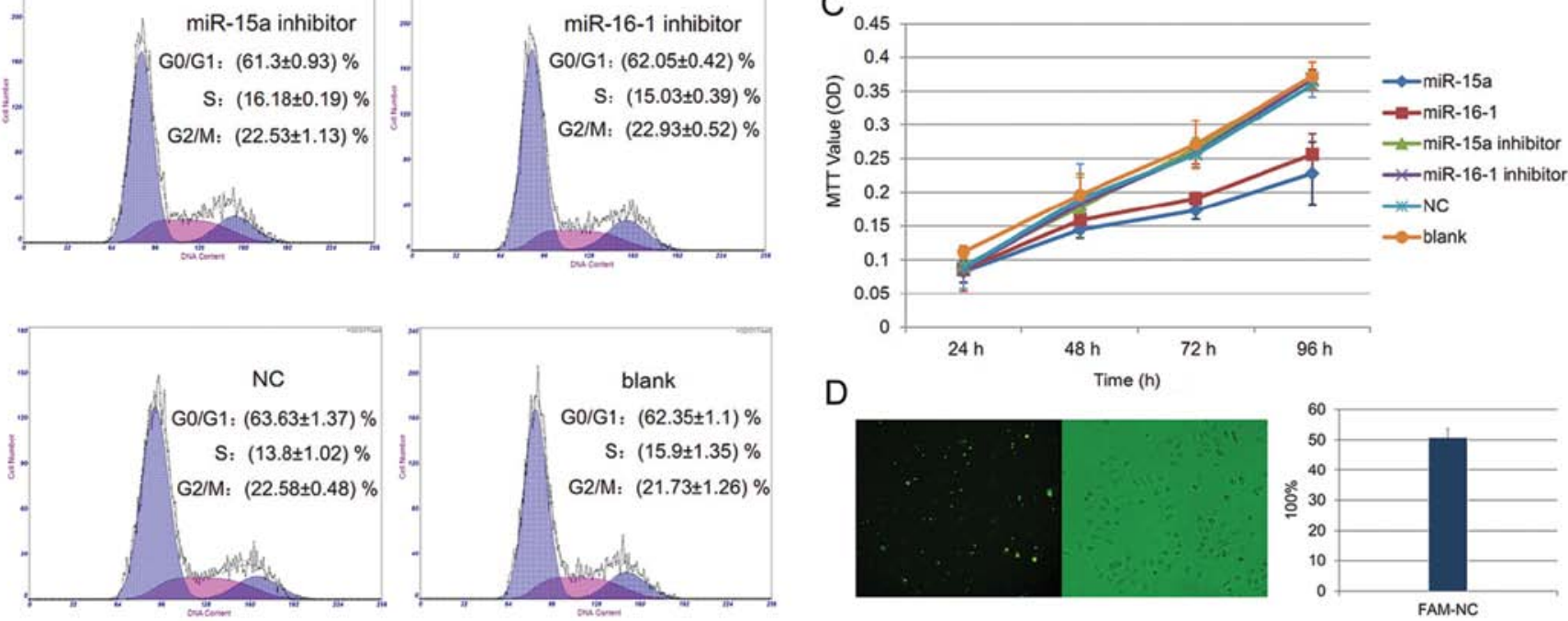

D
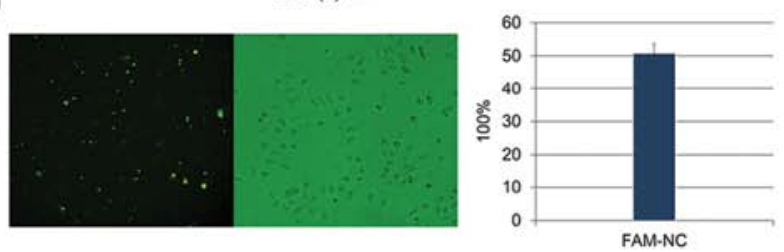

Figure 2. miR-15a and miR-16-1 induced cell cycle arrest and reduced proliferation. (A) Cell cycle analysis using flow cytometry. PI was used to mark the cell DNA and Annexin V was used to mark the phosphatidylserine translocation. The X-axis is the DNA content, whereas the Y-axis is the cell number. The data are the means $\pm \mathrm{SD}$ of four experiments evaluating the relative percentage of cells at $\mathrm{G} 0 / \mathrm{G} 1, \mathrm{~S}$, and $\mathrm{G} 2 / \mathrm{M}$ phase of the cell cycle. (B) At any given point in time, more cells are in the G0/G1 phase in the microRNAs treated cells than in the controls $(\mathrm{P}<0.05)$. (C) Cell growth detected by MTT. The data are the means \pm SD of eight independent experiments. The curves showed that cells transfected with miR-15a and miR-16-1 proliferate slower compared with the other groups $(\mathrm{P}<0.05)$. (D) The efficiency of transfection rate. The two graphs on the left showed pre- and post-transfection with 5'-FAM-marked negative control using Lipofectamine 2000. The table on the right shows the efficiency of transfection rate. The data are the means \pm SD of five independent experiments.

levels of apoptosis in the inhibitor, negative, and blank control groups (Fig. 1C left and D, P>0.05). Furthermore, converterPOD and haematoxylin staining demonstrated that there are more apoptotic cells in the experiment group compared with the other groups (Fig. 1C, right).

Alteration of cell cycle and cell proliferation by miR-15a and miR-16-1 in human osteosarcoma cell line SOSP-9607. Cell cycle analysis showed that more cells are in the G0/G1 phase in the microRNAs treated cells than in the controls (Fig. 2A and B, $\mathrm{P}<0.05)$. The MTT assay indicated that the proliferation curves of cells transfected with miR-15a and miR-16-1 are slower compared with that of the other groups. However, no differences are found compared with the inhibitor, negative control and blank groups, respectively (Fig. 2C, P>0.05).

miR-15a and miR-16-1 downregulate the protein levels of $C C N D 1$. After $48 \mathrm{~h}$ of transfection, the total RNA of each group was extracted and then used for RT-PCR. The results showed that miR-15a and miR-16-1 inhibit the mRNA levels of CCND1, but do not degrade them (Fig. 3A, P>0.05). Western blot was used to further confirm the post-transcriptional repression of CCND1. The results proved that miR-15a and miR-16-1 could downregulate the protein levels of CCND1 (Fig. 3B, $\mathrm{P}<0.05$ ).
Immunocytochemistry also showed that cells transfected with miR-15a and miR-16-1 have fewer CCND1 proteins (brown color) than the other groups (Fig. 3C).

miR-15a and miR-16-1 binding to the 3'UTR segment of CCND1. The results of the luciferase assays revealed that overexpression of miR-15a and miR-16-1 could reduce the luciferase activity from the segment containing CCND1-b (Fig. 4E left, $\mathrm{P}<0.05$ ), whereas CCND1-a has no effect on luciferase activity (Fig. 4E left, P>0.05). A control experiment with two types of mutated target mRNA sequences lacking seven $\left(3^{\prime} \mathrm{Ma}\right)$ or eight $\left(3^{\prime} \mathrm{Mb}\right)$ complementary bases of CCND1 cDNA was also performed. As expected, both mutants completely abolish the interaction between the two miRNAs and the 3'UTR of CCND1 (Fig. 4E right, $\mathrm{P}>0.05)$. The experimental data indicated that miR-15a and miR-16-1 regulate the expression of CCND1 by targeting the putative binding site CCND1-b.

\section{Discussion}

Various studies have shown the downregulation of miR-15a and miR-16-1 in osteosarcoma $(14,15)$. Moreover, these two miRNAs have been reported to induce apoptosis by targeting BCL-2 (16). Cimmino et al (17) showed that these 

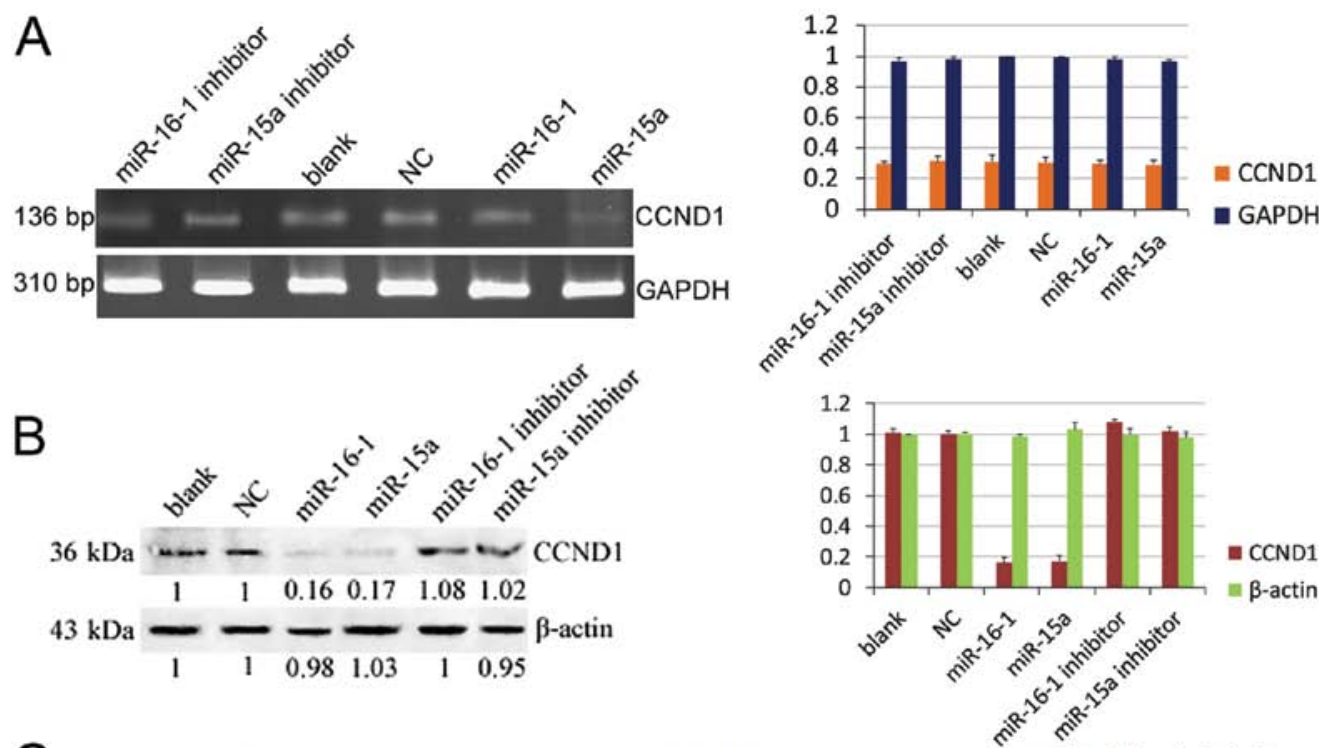

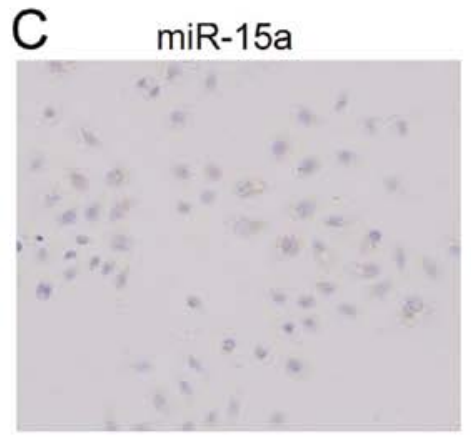

miR-16-1 inhibitor

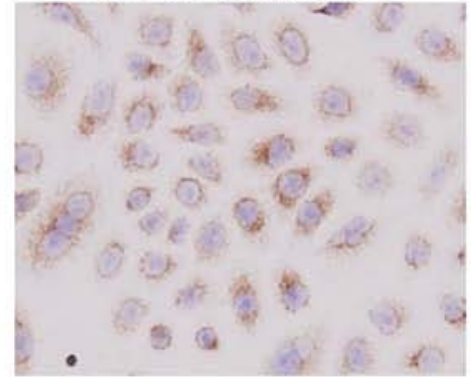

miR-16-1

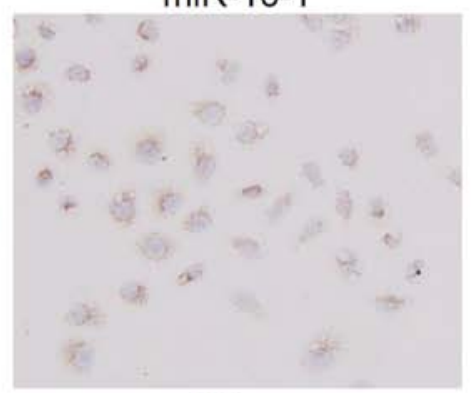

NC

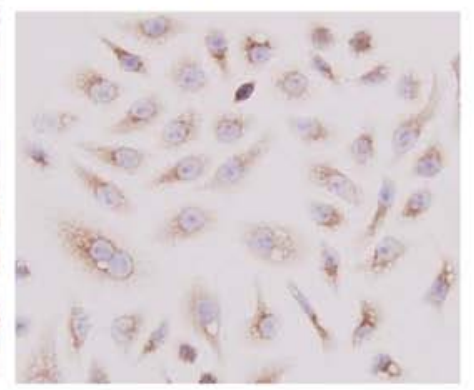

miR-15a inhibitor

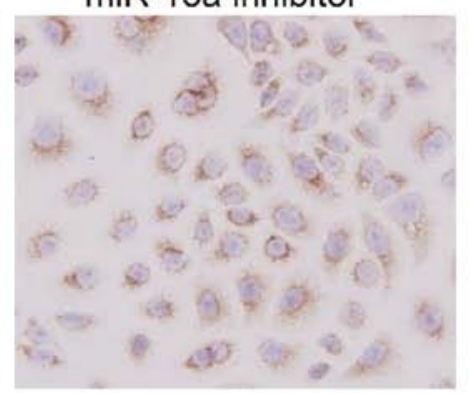

blank

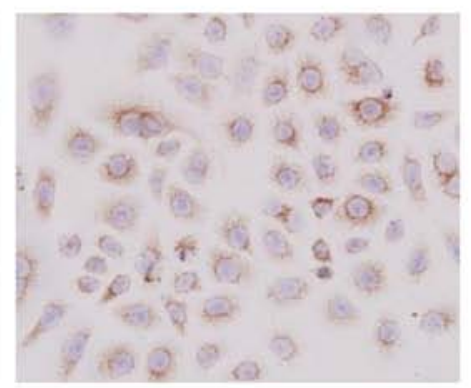

Figure 3. CCND1 is a target gene of miR-15a and miR-16-1. (A) Results of RT-PCR showed no difference on mRNA levels of CCND1 in each group (P>0.05). The production length of CCND1 and GAPDH are 136 and $310 \mathrm{bp}$, respectively. DL2000 was used as marker. (B) Western blot analysis of miR-15a/miR-16-1, miR-15a/miR-16-1 inhibitor, negative control, and blank control. The normalization was done using mouse monoclonal anti $\beta$-actin antibody. (C) Cyclin D1 immunocytochemistry staining of miR-15a, miR-16, miR-15a inhibitor, miR-16-1 inhibitor, negative control, and blank control. The brown color in the cytoplasm and nucleus are the locations of cyclin D1. The blue color in the nucleus is the staining of haematoxylin (magnification, x200).

microRNAs directly target the BCL-2 3'-untranslated region and significantly correlate with BCL-2 protein levels in CLL both in vitro and in vivo. Furthermore, BCL-2 protein levels are downregulated in response to miR-15a or miR-16-1 expression, leading to significant reduction in CLL cell proliferation. Lerner et al (18) demonstrated that putative tumor suppressor DLEU2 acts as a target gene of miR-15a and miR-16-1. DLEU2 overexpression blocks cellular proliferation and inhibits the colony-forming ability of tumor cell lines in a miR-15a/ miR-16-1-dependent manner. However, in the current study, RT-PCR did not show the BCL-2 mRNA expression in the SOSP-9607 cell line (Fig. 4B). This cell line was primarily cultured from the left tibia of a 17-year-old male patient (19-21). In the current study, miR-15a and miR-16-1 induced the apoptosis of SOSP-9607 cells, indicating that miR-15a and miR-16-1 induce apoptosis by targeting many genes. CCND1 was found through target screening and RT-PCR verification. This gene has been widely discussed due to its cell cycle functions. One of the aims of the current research is to investigate whether cyclin D1 correlates to the apoptosis induced by miR-15a and miR-16-1.

Cyclin D1 is an important regulator of cell cycle progression (22). It was cloned and identified from parathyroid adenoma by Motokura et al (23) in 1991. CCND1 is located in a cluster at 11 q13 with high percentage of G/C components. The 56-141 nucleotides sequence of cyclin D1 is a conserved sequence called 'cyclin box', which is the idio-combination areas to cyclin-dependent kinases (CDK). The G1/S phase transition 
A

Hsa-CCND1(a) 3'UTR: 1961 -CCAUUUUCUUAUUGCGCUGCUAC-1967 1111111

Hsa-miR-15a: GUGUUUGGUAAUACACGACGAU-5,

Hsa-miR-16-1:

III| |||||||

Hsa-CCND1(b)3'UTR: 2033-CUCUUUCACAUUGUUUGCUGCUA ${ }^{3}{ }^{\prime}{ }^{203}$

IIII 11111111

Hsa-miR-15a:

GUGUUUGGUAAUACACGACGAU-5'

Hsa-miR-16-1:

GCGGUUAUAAY, GACGACGAU-5,

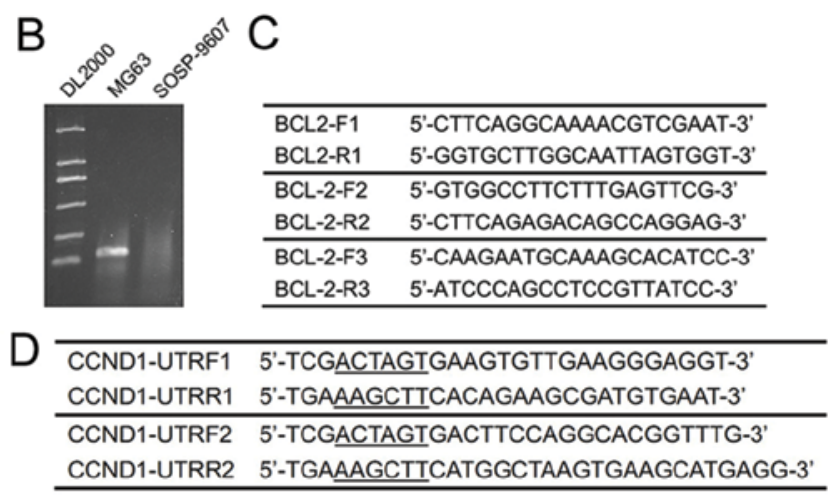

E

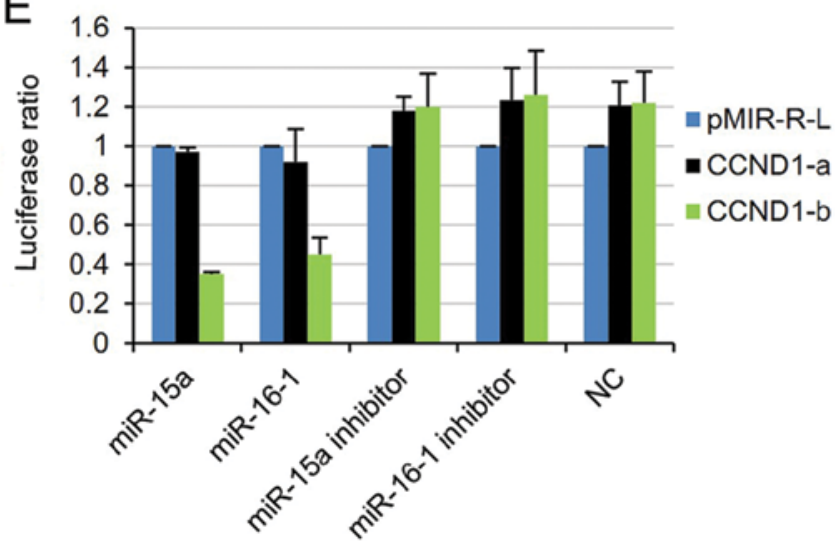

Figure 4. miR-15a and miR-16-1 may regulate the expression of CCND1 by targeting putative binding site CCND1-b. (A) Sites of complementarity sequences between microRNAs and CCND1 mRNA. (B) Using the first primer set of (C), MG63 has significant production length of 148 bp (middle), but SOSP-9607 has no product (right). The other two primer sets also showed the same results. (C) Three BCL-2 primer set segments used for RT-PCR. The production lengths are 148, 213, and 712 bp. (D) Two couples of primers designed for RT-PCR. The products were used for luciferase report. (E) Luciferase assays indicated that miR-15a and miR-16 downregulate the expression of CCND1 by targeting putative target site CCND1-b. Relative repression of the firefly Luciferase expression was standardized for transfection control, Renilla luciferase. PMIR-REPORT ${ }^{\mathrm{TM}}$ luciferase (pMIR-R-L, Promega) was used as empty vector. microRNA mimics, inhibitors, and negative control were used for transfections. Putative target segments CCND1-a, CCND1-b (Left), and mutant inserts CCND1-Ma and CCND1-Mb (Right) were used to construct luciferase reporter vector. All experiments were performed twice in triplicate (n=6).

is regulated primarily by D-type cyclins (D1, D2, or D3) belonging to the CDK4/CDK6 complex and the E-type cyclins (E1 or E2) of the CDK2 complex. These complexes cooperate in phosphorylating and preventing $\mathrm{pRB}$ binding to $\mathrm{E} 2 \mathrm{~F}$, thus activating E2F-mediated transcription and driving cells from G1 into $S$ phase (24).

Furthermore, various studies have reported that the E2F family can mediate apoptosis. For example, the ectopic expression of E2F1 leads to apoptosis in tissue-culture cells. Transgenic mice and E2F1 knockout mice develop tumors, partially due to suppressed apoptosis (25). Moreover, E2F1 and P53 cooperate to induce apoptosis $(26,27)$. Intensive research has identified several molecular mechanisms underlying this cooperation. First, E2F1 induces the stabilization and activation of P53. Second, E2F1 and P53 separately transactivate a plethora of crucial pro-apoptotic genes, raising the possibility that one or more of their respective targets cooperate to induce apoptosis. Third, several pro-apoptotic genes, including APAFI, SIVA, and the BH3 protein-encoding genes NOXA and PUMA, seem to be transcriptionally regulated by both E2F1 and P53 (28-33). Taken together with the activation of P53 by E2F1, this pattern of regulation constitutes a modified feed-forward loop that modulates E2F1 activity and apoptosis progression (34).

Activated CCND1 may have the ability to induce apoptosis through the cyclin D/E-CDK2/4/6-pRB-E2F1 pathway (Fig. 5).
In the current research, flow cytometry and TUNEL assay showed higher apoptosis ratios in experimental groups, demonstrating that miR-15a and miR-16-1 could induce apoptosis in osteosarcoma cells. From the cell cycle analysis, the experiment groups have longer G1 phase compared with the control groups, illustrating that miR-15a and miR-16-1 can induce cell cycle arrest. Moreover, the curve reflected that these two microRNAs could reduce cell proliferation. Thus, miR-15a and miR-16-1 induce apoptosis and cell cycle arrest in osteosarcoma.

The current study showed for the first time that miR-15a and miR-16-1 do not degrade the mRNA levels of CCND1, but regulate the expression of CCND1 by targeting putative binding site CCND1-b. Putting these findings together, it can be concluded that in the SOSP-9607 cell line, miR-15a and miR-16-1 induce apoptosis and cell cycle arrest by targeting CCND1. Nevertheless, miR-15a and miR-16-1 may have several target genes. During the experiment, other predicted target gene interference points should be avoided, a requirement which is both important and difficult. The data presented in the current research are of considerable therapeutic significance because miR-15 and miR-16-1 are natural anti-sense CCND1 interactors that could be used for therapy in tumor overexpressing CCND1.

In conclusion, the current experimental results reveal that miR-15a and miR-16-1 arrest cell cycle and induce apoptosis and negatively regulate CCND1 expression. These findings may 


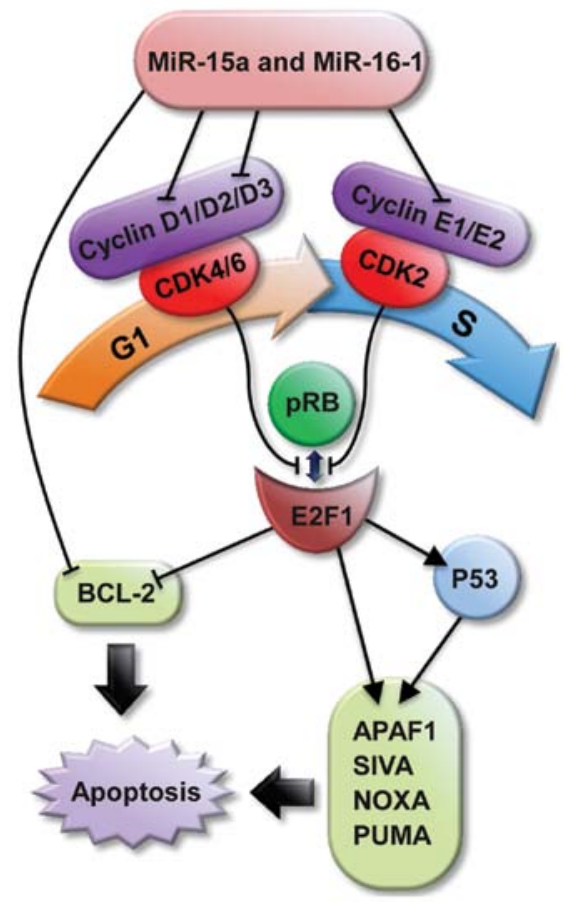

Figure 5. miR-15a and miR-16-1 induce apoptosis and cell cycle arrest through cyclin D/E-CDK2/4/6-pRB-E2F1 pathway. The G1/S phase transition is regulated primarily by D-type cyclins (D1, D2 or D3) in complex with CDK4/6, and E-type cyclins (E1 or E2) in complex with CDK2. These complexes cooperate in phosphorylating and preventing $\mathrm{pRB}$ binding to $\mathrm{E} 2 \mathrm{~F} 1$, thus activating E2F1-mediated transcription and driving cells from $\mathrm{G} 1$ into $\mathrm{S}$ phase. E2F-1 could upregulate P53, thus forming a modified feed-forward loop to activate many anti-oncogenes to induce apoptosis.

have important significance on the development of therapeutic approaches for osteosarcoma.

\section{Acknowledgements}

This study was supported by the Department of Orthopedic Surgery Center and Orthopedic Oncology Institute of People's Liberation Army and by the National Natural Science Foundation of China under Grant No. 81072194. We sincerely thank Jia-yong Fan for his assistance in polishing the standard of English in this manuscript.

\section{References}

1. Kosacka M, Piesiak P, Porebska I, et al: The cyclin A, B1, D1, and $\mathrm{E}$ expression in advanced non-small cell lung cancer - stages IIIB-IV (preliminary report). Pol Merkur Lekarski 30: 253-258, 2011 (in Polish).

2. Chen RW, Bemis LT, Amato CM, et al: Truncation in CCND1 mRNA alters miR-16-1 regulation in mantel cell lymphoma. Blood 112: 822-829, 2008.

3. Miska EA: How microRNAs control cell division, diffentiation and death. Curr Opin Genet Dev 15: 563-568, 2005.

4. Bartel DP: MicroRNAs: genomics, biogenesis, mechanism, and function. Cell 116: 281-297, 2004.

5. Carè A, Catalucci D, Felicetti F, et al: MicroRNA-133 controls cardiac hypertrophy. Nat Med 13: 613-618, 2007.

6. Bandi N, Zbinden S, Gugger M, et al: miR-15a and miR-16-1 are implicated in cell cycle regulation in a $\mathrm{Rb}$-dependent manner and are frequently deleted or down-regulated in non-small cell lung cancer. Cancer Res 69: 5553-5559, 2009.

7. Tsang WP and Kwok TT: Epigallocatechin gallate up-regulation of miR-16 and induction of apoptosis in human cancer cells. J Nutr Biochem 21: 140-146, 2010.
8. Guo CJ, Pan Q, Jiang B, Chen GY and Li DG: Effects of upregulated expression of microRNA-16 on biological properties of culture-activated hepatic stellate cells. Apoptosis 14: 1331-1340, 2009

9. Xu F, Zhang X, Lei Y, et al: Loss of repression of HuR translation by miR-16 may be responsible for the elevation of HuR in human breast carcinoma. J Cell Biochem 111: 727-734, 2010.

10. Bhattacharya R, Nicoloso M, Arvizo R, et al: MiR-15a and MiR-16 control Bmi-1 expression in ovarian cancer. Cancer Res 69: 9090-9095, 2009.

11. Bonci D, Coppola V, Musumeci M, et al: The miR-15a-miR-16-1 cluster controls prostate cancer by targeting multiple oncogenic activities. Nat Med 14: 1271-1277, 2008.

12. Xia L, Zhang DX, Du R, et al: miR-15b and miR-16-1 modulate multidrug resistance by targeting BCL2 in human gastric cancer cells. Int J Cancer 123: 372-379, 2008.

13. Bottoni A, Piccin D, Tagliati F, et al: miR-15a and miR-16-1 down-regulation in pituitary adenomas. J Cell Physiol 204: 280-285, 2005.

14. Nedelcu T, Kubista B, Koller A, et al: Livin and Bcl-2 expression in high-grade osteosarcoma. J Cancer Res Clin Oncol 134: 237-244, 2008.

15. Gao J, Yang TT, Qiu XC, et al: Cloning and identification of microRNA from human osteosarcoma cell line SOSP-9607. Ai Zheng 26: 561-565, 2007 (In Chinese).

16. Tsang TY, Tang WY, Chan JY, et al: P-glycoprotein enhances radiation-induced apoptotic cell death through the regulation of miR-16 and Bcl-2 expressions in hepatocellar carcinoma cells. Apoptosis 16: 524-535, 2011.

17. Cimmino A, Calin GA, Fabbri M, et al: miR-15 and miR-16 induce apoptosis by targeting BCL-2. Proc Natl Acad Sci USA 102: 13944-13949, 2005.

18. Lerner M, Harada M, Lovén J, et al: DLEU2, frequently deleted in malignancy, functions as a critical host gene of the cell cycle inhibitory microRNAs miR-15a and miR-16-1. Exp Cell Res 315: 2941-2952, 2009.

19. Shan LQ, Ma S, Qiu XC, et al: A novel recombinant immune-tBid with a furin site effectively suppresses the growth of HER2positive osteosarcoma cells in vitro. Oncol Rep 25: 325-331, 2011.

20. Wang LF, Zhou Y, Xu YM, et al: A caspase- 6 and anti-HER2 antibody chimeric tumor-targeted proapoptotic molecule decreased metastasis of human osteosarcoma. Cancer Invest 27: 774-780, 2009.

21. Chen X, Yang TT, Wang W, et al: Establishment and characterization of human osteosarcoma cell lines with different pulmonary metastatic potentials. Cytotechnology 61: 37-44, 2009.

22. Sherr CJ: D-type cyclins. Trends Biochem Sci 20: 187-190, 1995.

23. Motokura T, Bloom T, Kim HG, et al: A novel cyclin encoded by a bell-linked candidate oncogene. Nature 350: 512-515, 1991.

24. Liu Q, Fu H, Sun F, et al: miR-16-1 family induces cell cycle arrest by regulating multiple cell cycle genes. Nucleic Acids Res 36: 5391-5404, 2008.

25. Iaquinta PJ and Lees JA: Life and death decisions by the E2F transcription factors. Curr Opin Cell Biol 19: 649-657, 2007.

26. Wu X and Levine AJ: p53 and E2F-1 cooperate to mediate apoptosis. Proc Natl Acad Sci USA 91: 3602-3606, 1994.

27. Han EK, Ng SC, Arber N, Begemann M and Weinstein IB: Roles of cyclin D1 and related genes in growth inhibition, senescence and apoptosis. Apoptosis 4: 213-219, 1999.

28. Moroni MC, Hickman ES, Lazzerini Denchi E, et al: Apaf-1 is a transcriptional target for E2F and p53. Nat Cell Biol 3: 552-558, 2001.

29. Han J, Flemington C, Houghton AB, et al: Expression of bbc3, a pro-apoptotic BH3-only gene, is regulated by diverse cell death and survival signals. Proc Natl Acad Sci USA 98: 11318-11323, 2001.

30. Nakano K and Vousden KH: PUMA, a novel proapoptotic gene, is induced by p53. Mol Cell 7: 683-694, 2001.

31. Oda E, Ohki R, Murasawa H, et al: Noxa, a BH3-only member of the Bcl-2 family and candidate mediator of p53-induced apoptosis. Science 288: 1053-1058, 2000.

32. Fortin A, MacLaurin JG, Arbour N, et al: The proapoptotic gene SIVA is a direct transcriptional target for the tumor suppressors p53 and E2F1. J Biol Chem 279: 28706-28714, 2004.

33. Hershko T and Ginsberg D: Up-regulation of Bcl-2 homology 3 (BH3)-only proteins by E2F1 mediates apoptosis. J Biol Chem 279: 8627-8634, 2004.

34. Polager S and Ginsberg D: P53 and E2f: partners in life and death. Nat Rev Cancer 9: 738-748, 2009. 